\title{
Clinical Efficacy Evaluation of Er: YAG Laser Treatments for Obstructive Sleep Apnea Hypopnea Syndrome
}

\begin{abstract}
Jia Liu',", Jiuhui Yang,", , Min Zhang ${ }^{3}$, Yongjin Chen ${ }^{3}$ and Qiang $\mathrm{Li}^{3}$,
${ }^{1}$ State Key Laboratory of Military Stomatology \& National Clinical Research Center for Oral Diseases \& Shaanxi Clinical Research Center for Oral Diseases, Department of Orthodontics, School of Stomatology, China
\end{abstract}

${ }^{2}$ Department of Stomatology, 966 Hospital of PLA, China

${ }^{3}$ State Key Laboratory of Military Stomatology \& National Clinical Research Center for Oral Diseases \& Shaanxi International Joint Research Center for Oral Diseases, Department of General Dentistry \& Emergency, China

"Jia Liu and Jiuhui Yang contributed equally to this work

*Corresponding author's: Qiang Li, State Key Laboratory of Military Stomatology \& National Clinical Research Center for Oral Diseases \& Shaanxi International Joint Research Center for Oral Diseases, Department of General Dentistry \& Emergency, China

\section{ARTICLE INFO}

Received: 崫 August 01, 2019

Published: 幽 August 08, 2019

Citation: Jia Liu, Jiuhui Yang, Min Zhang, Yongjin Chen, Qiang Li. Clinical Efficacy Evaluation of Er: YAG Laser Treatments for Obstructive Sleep Apnea Hypopnea Syndrome. Biomed J Sci \& Tech Res 20(3)-2019. BJSTR. MS.ID.003447.

Keywords: Er: YAG laser; Obstructive Sleep Apnea Hypopnea Syndrome (OSAHS); Laser Therapy; Therapeutic Effect Evaluation; Follow up

\begin{abstract}
Obstructive sleep apnea hypopnea syndrome (OSAHS) has become an important public health concern. Although numerous treatments have been used, noninvasive and convenient methods are always desirable. This study aimed to evaluate the therapeutic effect of Er:YAG laser on OSAHS. Fifty-four mild OSAHS patients received Er:YAG laser therapy. The snoring severity and sleep quality were measured before the treatment, immediately after the 1st, 2nd and 3rd treatments, and after 3, 6 and 12 months. The patients' pain intensity, oropharyngeal feelings and satisfaction were recorded during the therapeutic period. We also measured the apnea hyponea index (AHI), average apnea duration and percentage of saturated oxygen $(\mathrm{SaO} \%)$ immediately after the completion of laser therapy and 12 months of follow-up with polysomnography (PSG). After Er:YAG laser therapy, the patients' snoring severity and sleep quality gradually improved over time compared with the pretreatment scores $(\mathrm{P}<0.05)$, and they were still lower than the original state until the end of the 12 months' follow-up $(\mathrm{P}<0.05)$. The pain score of the patients during the treatment period was low, and the oropharyngeal feelings were evidenced by throat dryness (approximately 90\%) and palatal paresthesia (12.96 $\sim 20.37 \%$ ). Additionlly, the patients showed well satisfaction with Er:YAG laser therapy. Moreover, the AHI and average apnea duration were lower after finishing the treatment and 12 months' follow-up than those of the pretreatment groups $(\mathrm{P}<0.05)$. These results showed us the Er:YAG laser could effectively improve the symptoms of OSAHS, indicating it as a minimally invasive method for alleviating OSAHS.
\end{abstract}

\section{Introduction}

Obstructive sleep apnea hypopnea syndrome (OSAHS) is characterized by recurrent episodes of partial or complete upper airway collapse during sleep that is highlighted by a reduction in or complete cessation of airflow despite documented going inspiratory efforts [1]. OSAHS is being increasingly identified as an important health issue. Additionally, this syndrome is considered an independent risk factor for hypertension, diabetes and cardiovascular diseases, leading to increased morbidity and mortality [2]. Although various treatments, such as continuous positive airway pressure [3] and uvulopalatopharyngoplasty [4] have been used to treat OSAHS, certain drawbacks, including foreign body sensation, discomfort and postoperative pain, still limit their popularization and application. Laser has been widely used in bio-medical field in recent years, and it attracts the great 
interests and attentions of many clinicians and researchers. The wavelength of the Er:YAG laser is $2940 \mathrm{~nm}$, and this wavelength determines its character to produce excellent energy absorption in water. In addition, Er:YAG laser has a limited depth of penetration through the tissue which exerts less damages to the adjacent treated areas and decreased erythema and edema [5]. Moreover, Er:YAG laser could has the potential to shrink the collagen and promote neocollagenesis [6,7]. All these merits make it an ideal laser for intraoral use [8], and scholars have tried to take advantage of the Er:YAG laser to treat snoring and have achieved positive effects recently [9-11]. However, except for snoring, which is the typical clinical manifestation of OSAHS, the patients often present with apnea, hypoventilation, and hypoxemia. Until now, few studies focus on the Er:YAG laser treatment of OSAHS, and there is still a lack of detailed clinical efficacy evaluations for this therapeutic method. Therefore, this study aimed to adopt an Er:YAG laser to treat OSAHS patients and conducted a preoperative and postoperative questionnaire survey and polysomnography measurements to understand and evaluate the clinical efficacy of the Er:YAG laser treatment for OSAHS.

\section{Materials and Methods}

\section{Study Population}

A total of 54 subjects ( 30 males and 24 females) with an average age of 47.6 years (range $=48-65$ years) were enrolled in the study. Adult subjects with mild OSAHS diagnosed by PSG with an apnea hypopnea index (AHI) of 5-15 and a body mass index (BMI) of less than 30 were recruited. Those individuals with the following characteristics, namely, younger than 18 , taking photosensitive drugs, pregnant, a nasal cavity obstruction (e.g., obvious deviation, polyps, adenoids, or turbinate hypertrophy), experiencing any oth- er surgical treatment that may affect snoring (e.g., tonsillectomy or nasal surgery), or have been diagnosed with central OSAHS, were excluded [10,11]. All subjects provided signed, informed consent prior to enrollment. The study was performed from December 2016 to March 2019. This investigation was approved by the Ethics Committee of School of Stomatology, The Fourth Military Medical University (IRB-REV-2016049), and the experiments were performed in accordance with the Declaration of Helsinki for humans.

\section{Measuring Equipment and Procedure}

All patients were treated with an Er:YAG laser (Fotona, Slovenia) using a PS03 handpiece. We used the laser parameters and the treatment protocol according to the manufacturer's instructions with a slight modification and performed the coverage of the defined regions using the so-called manual brushing technique and noncontact mode [9]. Specifically, the laser parameters were set as follows: linearly polarized mode; pulse width, $600 \mu \mathrm{m}$; spot diameter, $7 \mathrm{~mm}$; frequency, $10 \mathrm{~Hz}$; average output power, 5.2-6.9 Watt and energy density $1.3-1.8 \mathrm{~J} / \mathrm{cm}^{2}$, according to the participant's tolerance. The Er:YAG laser energy was applied to eight mouth and throat regions: the anterior pillar extending to the outer face up to the retromolar region and posterior third of the cheek (two symmetrical regions); the soft palate and uvula with the lower part of hard palate (two symmetrical regions); the posterior pillars and tonsils (two symmetrical regions); and the lateral and bottom sides of the tongue (two symmetrical regions) (Figure 1) [11]. Gag reflexes were overcome using relaxing breathing techniques, or a topical lidocaine spray. Every patient was treated three times (on the $1^{\text {st }}$ day ( $1^{\text {st }}$ Tre.), $15^{\text {th }}$ day ( 2 nd Tre.), and $45^{\text {th }}$ day ( $3^{\text {rd }}$ Tre.) $)$ with a total of 10000 to 14000 laser pulses delivered per session. The doctors, nurses and patients wore goggles to protect their eyes during the treatment.

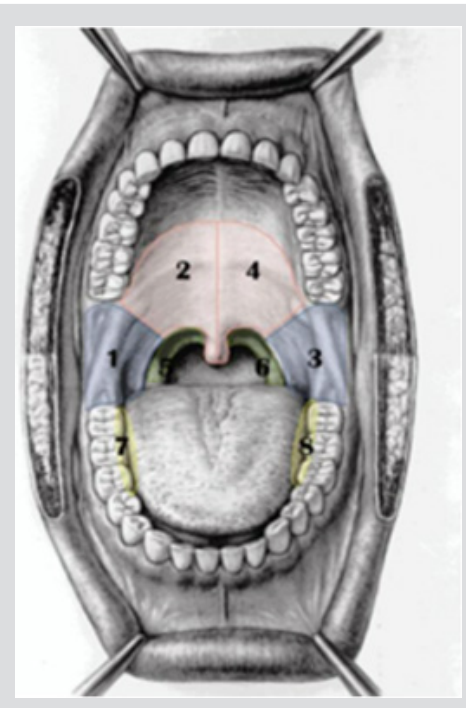

Figure 1: Schematic diagram of the Er:YAG laser irradiation area in the mouth. Regions 1 and 3: the anterior pillar extending to the outer face up to the retromolar region and posterior third of the cheek; Regions 2 and 4: the soft palate and uvula with the lower part of hard palate (two symmetrical regions); Regions 5 and 6: the posterior pillars and tonsils; and Regions 7 and 8: the lateral and bottom sides of the tongue (two symmetrical regions). 


\section{Evaluation Methodology}

Assessments were performed before the treatment (Pre. Tre.), post the $1^{\text {st }}$ treatment (Post $1^{\text {st }}$ Tre.), post the $2^{\text {nd }}$ treatment (Post $2^{\text {nd }}$ Tre.), post the $3^{\text {rd }}$ treatment (Post $3^{\text {rd }}$ Tre.), and during follow-ups at 3 months (follow-up $3 \mathrm{~m}$ ), 6 months (follow-up $6 \mathrm{~m}$ ) and 12 months (follow-up $12 \mathrm{~m}$ ). At each time point, the patients' bed partners were interviewed regarding the patients' snoring severity with an eleven-point scale (0-10), and the patients' sleep quality were evaluated by the Pittsburgh sleep quality index (PSQI) [12] and Epworth Sleepiness Scale (ESS) [13]. In addition, immediately after each treatment session, the pain response was measured on a visual analog scale (VAS) (from $0=$ no pain to $10=$ very severe pain) [14], a questionnaire was used to evaluate the subjective satisfaction on a scale of $0-3(0=$ not satisfied; $1=$ somewhat satisfied; $2=$ satisfied; $3=$ very satisfied), and the oropharyngeal sensations, such as pharyngeal dryness and palatal paresthesia, were recorded at the

\section{Results}

\section{Intraoral View}

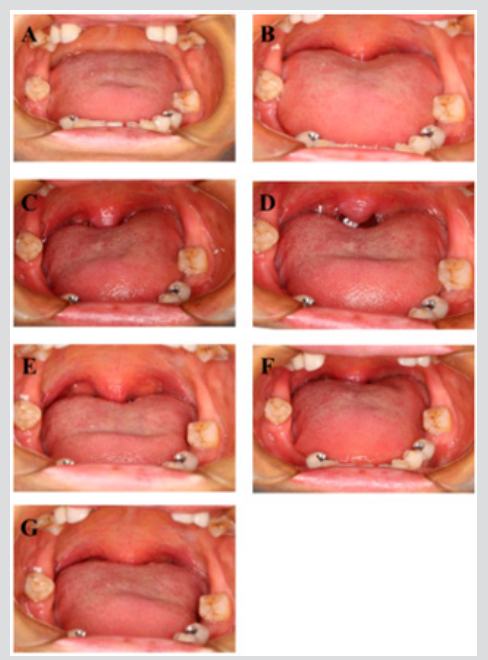

Figure 2: Typical clinical case treated by the Er:YAG laser. A, intraoral view of Pre. Tre. B, intraoral view of post $1^{\text {st }}$ Tre. C, intraoral view of post $2^{\text {nd }}$ Tre. $D$, intraoral view of post $3^{\text {rd }}$ Tre. E, intraoral view of follow-up $3 \mathrm{~m}$. F, intraoral view of follow-up $6 \mathrm{~m}$. G, intraoral view of follow-up $12 \mathrm{~m}$.

Typical clinical case is presented in Figure 2. The airway of the OSAHS patient was almost completely obstructed before the Er:YAG laser treatment (Figure 2A). After the $1^{\text {st }}$ Tre., the base of uvula could be observed (Figure $2 \mathrm{~B}$ ). After the $2^{\text {nd }}$ Tre., the whole uvula and palatoglossal arch showed up (Figure 2C). When the $3^{\text {rd }}$ Tre. was completed, the uvula, palatoglossal arch, tonsil and even posteior pharyngeal wall were revealed (Figure 2D). Although the pharyngeal cavity narrowed gradually during the follow-up period, partial uvula and palatoglossal arch still showed up at end ((Figure 2E, F, G).

\section{Snoring Severity}

The mean snoring severity score gradually decreased after each Er:YAG laser treatment session and was lower than that of the Pre. Tre. score (all $\mathrm{P}<0.05$ ). The score of Post $3^{\text {rd }}$ Tre. was lower than the same time. Moreover, we collected and compared the AHI, average apnea duration, and percentage of saturated oxygen (SaO\%) of 49 patients before and after Er:YAG laser therapy and 12 months of follow-up (5 patients disagreed to receive PSG reexamination after treatment or during follow-up because of the cost or time).

\section{Statistical Analysis}

All the data were analyzed with SPSS 17.0 software (SPSS Inc., Chicago, IL, USA). The Chi-square test and one-way analysis of variance were used to test the gender consistency and age consistency of patients, respectively. The homogeneity of variance of all the recorded data was tested by the Bartlet $t$ test. The results of measurement data were expressed as the mean \pm standard deviation (S.D.), and the Bonferroni test was used for comparison among the groups, while the results of counting data were expressed by rate (\%), and comparison among groups was conducted by the $\chi 2$ test. The statistical significance was set at $\mathrm{p}<0.05$. 


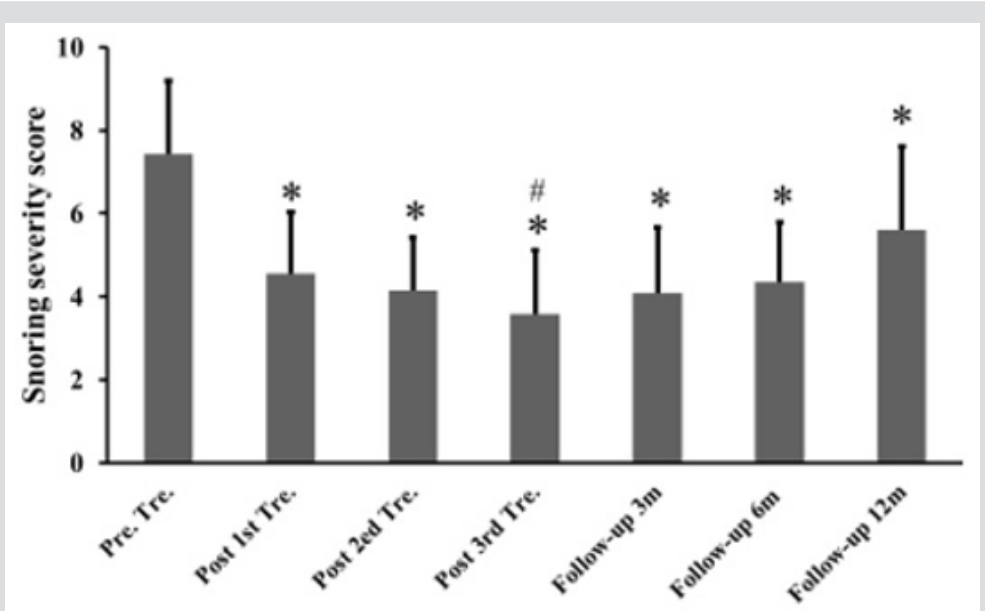

Figure 3: The snoring severity of obstructive sleep apnea hypopnea syndrome (OSAHS) patients treated with the Er:YAG laser. Data were expressed as mean $\pm S$.D. ${ }^{*} \mathrm{P}<0.05$ vs. Pre. Tre. and \# $\mathrm{P}<0.05$ vs. Post $1^{\text {st }}$ Tre. (Bonferroni test ).
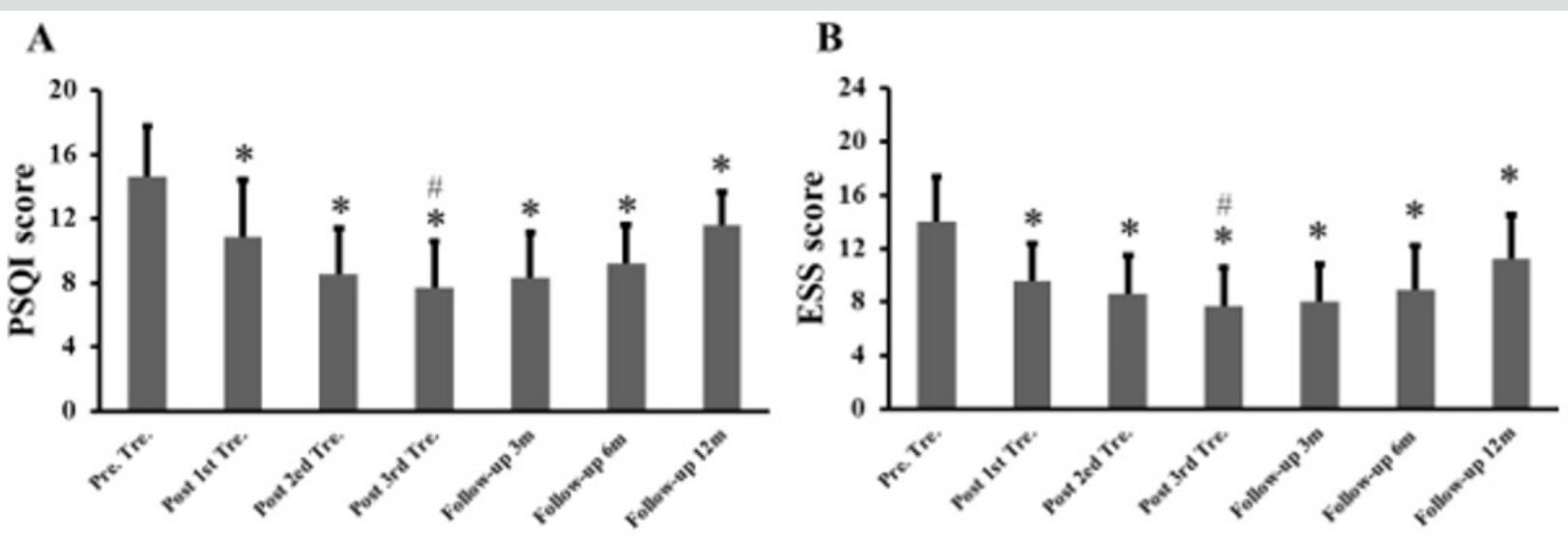

Figure 4: The sleep disorder status of OSAHS patients treated with the Er:YAG laser. A. The Pittsburgh sleep quality index (PSQI) score of OSAHS patients treated with the Er:YAG laser. B, The Epworth sleepiness scale (ESS) score of OSAHS patients treated with the Er:YAG laser. Data were expressed as mean \pm S.D. * $\mathrm{P}<0.05$ vs. Pre. Tre. and \# P<0.05 vs. Post 1 st Tre. (Bonferroni test).

\section{Pain Response}

The VAS measurements showed that the pain degree scores after each Er:YAG laser treatment session were approximately 4 , and no significant differences were found among the treatments $(\mathrm{P}=0.476)$.

\section{Oropharyngeal Feeling}

Based on the interview results, pharyngeal dryness existed in approximately $90 \%$ of patients during each Er:YAG treatment session. The occurrence of palatal paresthesia was between Table 1: The oropharyngeal feeling of obstructive sleep apnea hypopnea syndrome (OSAHS) patients treated with Er:YAG laser.
patient) reported being not satisfied with the treatment (Table 2).

\begin{tabular}{|c|c|c|c|c|}
\hline \multirow{2}{*}{} & \multicolumn{2}{|c|}{ Pharyngeal dryness [n(\%)] } & \multicolumn{2}{|c|}{ Palatal paresthesia [n(\%)] } \\
\cline { 2 - 5 } & Yes & No & $11(20.37 \%)$ & $43(79.63 \%)$ \\
\hline $1^{\text {st }}$ Tre. & $48(88.89 \%)$ & $6(11.11 \%)$ & $11(20.37 \%)$ & $43(79.63 \%)$ \\
\hline $2^{\text {nd }}$ Tre. & $49(90.74 \%)$ & $5(9.26 \%)$ & $7(12.96 \%)$ & $47(87.04 \%)$ \\
\hline $3^{\text {rd }}$ Tre. & $48(88.89 \%)$ & $6(11.11 \%)$ & Yes & 4 \\
\hline
\end{tabular}

$12.96 \%$ and $20.37 \%$, with the main symptoms as "burning feeling", "acmesthesia" or "ant crawling" (Table 1). All these oropharyngeal feelings disappeared within the next day or two.

\section{Satisfaction Evaluation}

The patients showed good satisfaction after each Er:YAG laser treatment with an increasing trend. Of all the patients, when the satisfied, 55.56\% (30 patients) that they were satisfied, 14.81\% (8 patients) reported being somewhat satisfied, and only 5.56\% (3 therapy was finished, $24.07 \%$ (13 patients) said that they were very 
Table 2: The oropharyngeal feeling of obstructive sleep apnea hypopnea syndrome (OSAHS) patients treated with Er:YAG laser.

\begin{tabular}{|c|c|c|c|c|}
\hline & Not satisfied & Somewhat satisfied & Satisfied & Very satisfied \\
\hline $1^{\text {st }}$ Tre. & $7(12.96 \%)$ & $16(29.63 \%)$ & $20(37.04 \%)$ & $11(20.37 \%)$ \\
\hline $2^{\text {nd }}$ Tre. & $2(3.70 \%)$ & $15(27.78 \%)$ & $28(51.85 \%)$ & $9(16.67 \%)$ \\
\hline $3^{\text {rd }}$ Tre. & $3(5.56 \%)$ & $8(14.81 \%)$ & $30(55.56 \%)$ & $13(24.07 \%)$ \\
\hline
\end{tabular}

\section{PSG Test}

Of the 54 OSAHS patients, 49 patients finished testing their sleep situation via PSG before, after and follow-up with the Er:YAG laser treatment. In those patients who underwent the PSG measurement three times, the AHI was $12.2 \pm 2.1 / \mathrm{h}$ (before), $2.7 \pm 1.1 / \mathrm{h}$ (after) and $5.4 \pm 2.5 / \mathrm{h}$ (follow-up) (Figure $5 \mathrm{~A}$ ), and the average apnea durations were $20.5 \pm 6.3 \mathrm{~s} / \mathrm{h}$ (before), $12.3 \pm 4.4 \mathrm{~s} / \mathrm{h}$ (after) and $16.6 \pm 4.7 \mathrm{~s} / \mathrm{h}$ (follow-up) (Figure 5B). Both indices showed significant differences among the three tests $(\mathrm{P}<0.05)$. There were no differences for that of $\mathrm{SaO} \%$ when compared with the three groups $(96.9 \pm 0.9 \%$ (before), 97.0 $\pm 0.9 \%$ (after) and $96.8 \pm 0.8 \%$ (follow-up), $\mathrm{P}>0.05$ ) (Figure 5C).
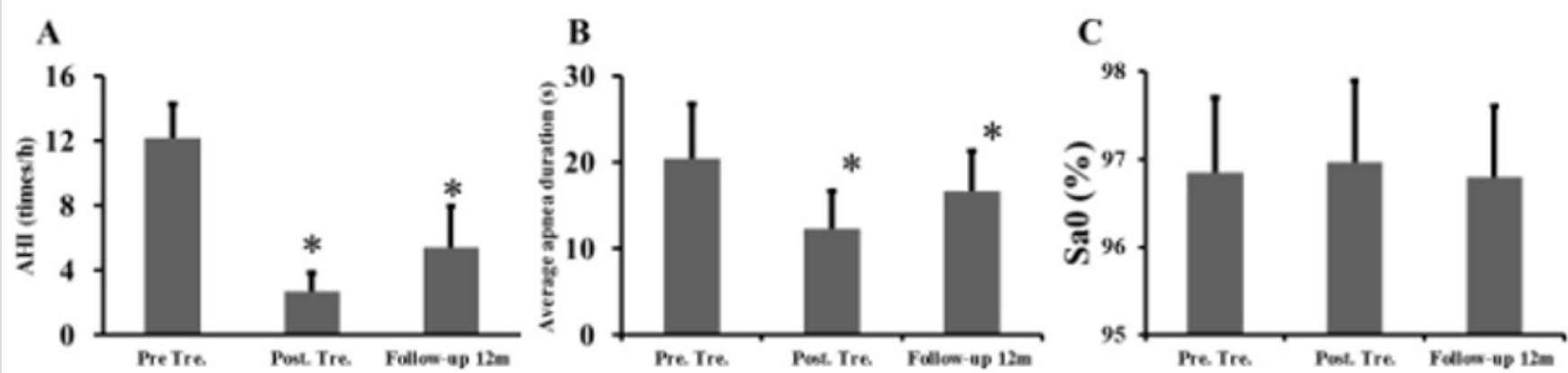

Figure 5: The measurement indices obtained from the polysomnography (PSG) test. A, The apnea hyponea index (AHI); B, The average apnea duration; $\mathrm{C}$, the percentage of saturated oxygen $(\mathrm{SaO} \%)$. Data were expressed as mean \pm S.D. ${ }^{*} \mathrm{P}<0.05$ vs. Pre. Tre. (Bonferroni test).

\section{Discussion}

Since the concept of OSAHS was put forward by American scholar Guileminault in 1973 [15], people have paid increasingly more attention to it, and related research on the disease has gradually deepened. Researchers have recognized OSAHS as a systemic disease rather than merely snoring and apnea $[16,17]$. Long-term chronic hypoxia due to OSAHS is harmful to the heart, brain, kidney, and other systemic organs and is even associated with increased mortality and morbidity [18].

Although the pathogenesis of OSAHS has not been fully understood, the direct cause is clear that stenosis and obstruction occur in the upper airway. As a consequence, the therapeutic methods of OSAHS mainly focus on symptomatic treatments, including nonsurgical procedures such as wearing an oral appliance or a continuous positive airway pressure device $[19,20]$, and surgical treatment for most severe OSAHS patients [21,22]. However, because of the inconvenience and invasiveness of these treatments, patients and physicians are still seeking OSAHS treatment methods that cause less damage and better effects. In recent years, some dentists proposed the idea of alleviating snoring with an Er:YAG laser and proved it to be a new, minimally invasive and painless treatment after conducting tentative studies [9-11]. The laser used in this study is the lattice Er:YAG laser. This laser could split the water molecules to produce oxygen free radicals after being absorbed by the water molecules in the oral mucosa, stimulate fibroblasts to secrete collagen and extracellular matrix [23-25], and result in the contracture and remodeling of collagen fibers in the mucosal lamina propria of the treated area [5]. The tissue contraction effects of Er:YAG laser in this study were obvious as it gradually opened the patients' obstructed airway time and time again evidenced by the tightened uvula, soft palate and surrounding tissues. A recent study demonstrated that the oropharyngeal airway volume significantly increased after the treatment as a result of the photothermic effects of the Er:YAG lasers [26].

In the present study, the average score of snoring severity after each Er:YAG laser treatment session was not only lower than that before treatment but also showed a gradually declining trend. Moreover, compared with the patients' initial situations, the PSQI results showed obviously improved sleep quality, and the ESS results showed significantly relieved daytime sleepiness after receiving laser therapy. The above findings demonstrated that Er:YAG treatment not only reduced the snoring symptoms but also improved the sleep quality in the patient. An earlier study [27] involving 21 patients treated by Er:YAG laser indicated an average snoring reduction after the first session between 30-60\% (on average approximately 43\%) and after the second session between $50-90 \%$ (average 62\%). Additionally, the improvement in the total score of the questionnaire after the first session increased approximately $45 \%$, while the improvement increased approximately 68\% after the second session. Another study [9] measured the average snoring 
severity score and average sleep disordered breathing score and showed similar findings with evidence that the former index before and at the first two follow-ups (at 14 and 45 days) improved by $50.5 \%$, while the latter index improved by $46 \%$. In addition, after comparing the degree of snoring severity and sleep disturbance among the three treatments, the scores after the third treatment session were lower than those after the first treatment session but not different from those after the second treatment session. These results suggest that the former two treatments were more effective in improving OSAHS symptoms, while the third treatment mainly played a role in maintaining and consolidating the therapeutic effects.

We investigated patient satisfaction after each Er:YAG laser treatment, and the results showed good satisfaction. Specifically, the proportion of satisfied and very satisfied patients increased with the progress of treatment. The satisfaction rate of the latter two treatments reached over $90 \%$ when the treatment was completed, indicating that the patients demonstrated good acceptance for the minimally invasive Er:YAG laser treatment of OSAHS. This laser therapy is non-ablative, and the mechanism of action is a photothermal effect causing heating of the treated areas to a well-controlled temperature [9]. A clinical study has proven that the scanning irradiation with an Er:YAG laser does not burn the mucosa tissue in the oropharynx because the temperature of the treated area was $41.5^{\circ} \mathrm{C}$ [10]. In another animal study, no exposed wound, bleeding, or macroscopic necrosis were observed in the Er:YAG laser irradiation area after sacrificing the animals, and the researchers noted a submucosal thermal effect at a depth of 0.4 $\mathrm{mm}$ with most of the epithelial tissue preserved histopathological [28]. This effect may be the reason for the lower incidence of palatal paresthesia and less pain in patients after being treated with OSAHS by Er:YAG laser in the present study. The pharyngeal dryness in most patients may be related to the absorption of laser heat by water in the mucosa as well as the loss of water caused by the mouth being open for a long time.

In our study, patients were followed up with for 12 months. Based on the subjective measures of the snoring severity and sleep disturbance (PSQI and ESS tests), the efficacy of Er:YAG laser therapy for OSHAS could be maintained for 12 months despite a decreasing trend with time. In addition, the AHI, average apnea durations and $\mathrm{SaO} \%$ were measured via the PSG test for 49 patients. Except for the SaO\%, the other two observation indices significantly decreased when Er:YAG laser therapy was finished and were sustained until the end of the follow-up in spite of the narrowed pharyngeal cavity. Mirackial \& Vizintin [9] stated that the achieved improvement for the majority of patients $(62.5 \%)$ was sustainable or was still improving at 6 months after the laser treatment, and the percentage of the still sustainable effect at 1215 months was $31.3 \%$. Storchi et al. reported that nearly $2 / 3$ of the patients $(71.9 \%)$ affirmed that their improvement was stable after 5 to 24 months (median 18.5 months) [11]. Combined with our research, it is obvious that the effect of Er:YAG laser therapy on OSHAS is stable and persistent. The result of unchanged $\mathrm{SaO} \%$ in the present study is consistent with a previous study that showed no significant difference in the amount of oxygen in the blood before and after laser treatment [29].

There are still some limitations in this study that need to be addressed. In the future, we need to further expand the sample size. Additionally, factors such as the anatomical morphology of the upper airway, the collagen remodeling ability of the pharynx, and the gender and age of the patient should be taken into consideration. Moreover, the selection of laser parameters for specific clinical indications and longer follow-ups need to be further explored and clarified in future experiments. In conclusion, this study indicates that Er:YAG laser treatment is a safe and very successful method for reducing snoring and sleep apnea and effectively improves the symptoms of patients diagnosed with mild OSAHS with slight side effects and high satisfaction. Obviously, OSAHS treatment by Er:YAG laser has promising application prospects.

\section{Acknowledgments}

The work was supported by the National Nature Science Foundation of China (No. 81500851and 81701002) and the Independent Research Project of State Key Laboratory of Military Stomatology (No. 2017ZB05). These funding sources were not involved in any part of the study, the writing of the manuscript, or in the decision to submit the manuscript for publication.

\section{Conflicts of Interest}

The authors declare that there are no conflicts of interest regarding the publication of this paper.

\section{Author Contributions}

Qiang Li, conception and study design, and critical revision of the manuscript; Jia Liu and Jiu-hui Yang, data collection and analysis as well as manuscript preparation; Min Zhang and Yong-jin Chen, results interpretation. All authors approved this edited version of article to be published.

\section{References}

1. De Backer W (2013) Obstructive sleep apnea/hypopnea syndrome. Panminerva Med 55(2): 191-195.

2. Lacedonia D, Carpagnano GE, Sabato R, Storto MM, Palmiotti GA, et al. (2016) Characterization of obstructive sleep apnea-hypopnea syndrome (OSA) population by means of cluster analysis. J Sleep Res 25(6): 724730

3. Chen X, Niu X, Xiao Y, Dong J, Lu M, et al. (2015) Effect of continuous positive airway pressure on leptin levels in patients with obstructive sleep apnea: a meta-analysis. Otolaryngol Head Neck Surg 152(4): 610618.

4. Choi JH, Cho SH, Kim SN, Suh JD, Cho JH (2016) Predicting Outcomes after Uvulopalatopharyngoplasty for Adult Obstructive Sleep Apnea: A Meta-analysis. Otolaryngol Head Neck Surg 155(6): 904-913.

5. Tian HS, Lin DP, Zhao TY, Yu B (2015) Histological change of rabbit oral mucosa after fractional Er:YAG laser irradiation. Chi J Oral Max Sur 13(2): 117-123. 
6. Wanitphakdeedecha R, Meeprathom W, Manuskiatti W (2017) A pilot study of treatment of striae distensae with variable square pulse Erbium: YAG laser resurfacing. J Cosmet Dermatol 16(4): 466-470.

7. Yang J, Wang S, Dong L, An X, Li Y, et al. (2016) Skin healing and collagen changes of rats after fractional erbium:yttrium aluminum garnet laser: observation by reflectance confocal microscopy with confirmed histological evidence. Lasers Med Sci 31(6): 1251-1260.

8. Wlodawsky RN, Strauss RA (2004) Intraoral laser surgery. Oral Maxillofac Surg Clin North Am 16(2): 149-163.

9. Mirackial K, Vizintin Z (2013) Nonsurgical Minimally Invasive Er:YAG Laser Snoring Treatment. J LA\&HA 1: 36-41.

10. Cetinkaya EA, Turker M, Kiraz K, Gulkesen HK (2016) Er:Yag Laser Treatment of Simple Snorers in an Outpatient Setting. ORL J Otorhinolaryngol Relat Spec 78(2): 70-76.

11. Storchi IF, Parker S, Bovis F, Benedicenti S, Amaroli A (2018) Outpatient erbium:YAG (2940 $\mathrm{nm}$ ) laser treatment for snoring: a prospective study on 40 patients. Lasers Med Sci 33(2): 399-406.

12. Buysse DJ, Monk TH, Berman SR, Kupfer DJ (1989) The Pittsburgh Sleep Quality Index: a new instrument for psychiatric practice and research. Psychiatry Res 28(2): 193-213.

13. Johns MW (1991) A new method for measuring daytime sleepiness: the Epworth sleepiness scale. Sleep 14: 540-545.

14. Price DD, McGrath PA, Rafii A, Buckingham B (1983) The validation of visual analogue scales as ratio scale measures for chronic and experimental pain. Pain 17(1): 45-56.

15. Guilleminault C, Eldridge FL, Dement WC (1973) Insomnia with sleep apnea: a new syndrome. Science 181(4102): 856-858.

16. Zamarron C, García Paz V, Riveiro A (2008) Obstructive sleep apnea syndrome is a systemic disease. Current evidence. Eur J Intern Med 19(6): 390-398.

17. Wang Q, Zhang C, Jia P, Zhang J, Feng L, et al. (2014) The association between the phenotype of excessive daytime sleepiness and blood pressure in patients with obstructive sleep apnea-hypopnea syndrome Int J Med Sci 11(7): 713-720.

\section{ISSN: 2574-1241}

DOI: 10.26717/BJSTR.2019.20.003447

Qiang Li. Biomed J Sci \& Tech Res

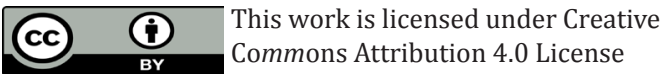

Submission Link: https://biomedres.us/submit-manuscript.php
18. Kaw R (2015) Obstructive sleep apnea in US veterans was associated with increased mortality and morbidity. Ann Intern Med 163: JC12.

19. Marklund M (2017) Update on Oral Appliance Therapy for OSA. Curr Sleep Med Rep 3(3): 143-151.

20. Jiang YQ, Xue JS, Xu J, Zhou ZX, Ji YL (2017) Efficacy of continuous positive airway pressure treatment in treating obstructive sleep apnea hypopnea syndrome associated with carotid arteriosclerosis. Exp Ther Med 14(6): 6176-6182.

21. Stuck BA, Ravesloot MJL, Eschenhagen T, de Vet HCW, Sommer JU (2018) Uvulopalatopharyngoplasty with or without tonsillectomy in the treatment of adult obstructive sleep apnea - A systematic review. Sleep Medicine 50: 152-165.

22. Pang KP, Siow JK (2009) Sutter bipolar radiofrequency volumetric tissue reduction of palate for snoring and mild obstructive sleep apnoea: is one treatment adequate? J Laryngol Otol 123(7): 750-754.

23. Majaron B, Srinivas SM, Huang He, Nelson JS (2000) Deep coagulation of dermal collagen with repetitive Er:YAG laser irradiation. Lasers Surg Med 26(2): 215-222.

24. Drnovsek-Olup B, Beltram M, Pizem J (2004) Repetitive Er:YAG laser irradiation of human skin: a histological evaluation. Lasers Surg Med 35(2): 146-151.

25. Beltram M, Zivin M, Drnovsek B (2010) Collagen synthesis after laser skin resurfacing of the periocular skin. Zdrav.Vestn 79: 111-116.

26. Lee CYS (2015) Evaluation of a non-ablative Er: YAG laser procedure to increase the oropharyngeal airway volume: a pilot study. Dental, Oral and Cranio Res 1(3): 56-59.

27. Jugoslav J (2011) NightLaseTM-Laser-Assisted Snoring and Apnea Reduction, 9 Months of Experience. J LA\&HA 201: S11.

28. Unver T, Aytugar E, Ozturan O, Kıran T, Ademci E, et al. (2016) Histological Effects of Er:YAG Laser Irradiation with Snoring Handpiece in the Rat Soft Palate. Photomed Laser Surg 34(8): 321-325.

29. Svahnström K (2013) Er:YAG Laser Treatment of Sleep-Disordered Breathing. J LA\&HA 2: 13-16. 